\title{
Snacking habits, speed of eating and their associations with body mass index
}

\author{
H. Young and B. Ratcliffe \\ Robert Gordon University, St Andrew Street, Aberdeen, AB25 1HG, UK
}

Snacking and irregular meal patterns have been implicated in the development of overweight and obesity ${ }^{(1,2)}$. However, there is conflicting evidence such as that from NHANES that snacking reduces the risk of adolescents being overweight ${ }^{(3)}$. Eating quickly has also been implicated in the overconsumption of dietary energy, and associated with higher body mass index (BMI) ${ }^{(4)}$. Both meal pattern and speed of eating may be amenable to intervention to prevent obesity so it is important to clarify the effects of these on energy intake and BMI.

The aims of this study were to explore the relationships between self-reported BMI and snacking habits and speed of eating in young adults. A questionnaire was distributed at two university premises for self-completion by subjects. Questions explored subjects' characteristics, aspects of meal habits including the meal time environment, eating patterns, the number of people present during a typical meal, reasons for snacking between meals, and speed of eating. The word 'snack' was not used in the questionnaire to avoid the ambiguities and lack of clarity in its use ${ }^{(5)}$. Drop boxes were used to preserve the anonymity of the respondents.

The questionnaires that were returned $(n$ 186) were coded and statistically analysed using SPSS Windows version 17 . The respondents were $32 \%$ ( $n$ 59) males and 68\% ( $n$ 127) females, 96\% ( $n$ 178) were students, and 88\% ( $n$ 164) were between 18-24 years of age. Analysis of self-reported data revealed that subjects had a mean weight of 68.3 (SD 14.6) kg, a mean height of 1.70 (SD 0.10 ) m, and a mean BMI of 23.4 (SD 4.2) kg/m². A majority of $84 \%$ ( $n$ 156) reported eating between meals and there was no significant effect of gender for this. However, this was inconsistent across the categories of BMI with $73 \%$ of those in the ideal BMI range reporting this compared with $30 \%$ of those who were overweight. Those who ate between meals were more likely to have a BMI in the normal range refuting the idea that people who snack are more likely to be overweight. There was a positive correlation between the number of days respondents had lunch and BMI $(P=0.034)$ perhaps indicating that missing this meal is used in weight control. There was no other association for meals and snacks at any other time of the day and BMI. There was no significant effect of meal time environment or meal pattern during a typical day on BMI.

Males were faster eaters than females $(P=0.005)$. A positive correlation $(P=0.024)$ was found between self-reported speed of eating and BMI in these young adults and this is similar to the findings in middle-aged Japanese that fast eaters were more likely to be overweight $^{(4)}$. In response to questions about factors affecting speed of eating, $80 \%(n 149)$ indicated that they ate faster when hungry, $68 \%$ ( $n$ 126) indicated that they ate faster when busy, and $70 \%$ ( $n$ 130) that they ate slower in company.

It should be noted that this study included a small sample population. The reliability and potential bias of using self-reporting of weight, height, and speed of eating should be considered when interpreting these results. Nevertheless, it is concluded that there is evidence for an association between speed of eating and BMI in young adults and the findings warrant further investigation and provide some indication of behaviours that could be modified to reduce the risk of becoming overweight or obese.

1. De Graaf C (2006) Appetite 47, 18-23.

2. Bes-Rastrollo M, Sanchez-Villegas A, Basterra-Gortari FJ et al. (2009) Clinical Nutr 29, 323-330.

3. Keast DR, Nicklas T \& O'Neil C (2010) Am J Clin Nutr 92, 428-435.

4. Maruyama K, Sato S, Ohira T et al. (2008) BMJ 337, a2002.

5. Chamontin A, Pretzer G \& Booth DA (2003) Appetite 41, 21-29. 